\title{
IoT based Road Traffic Management System for Metropolitan Cities
}

\author{
Chandrakala Arya, Manoj Diwakar, KajalAggarwal
}

\begin{abstract}
Many cities in the world face jamming problems in road traffic, particularly in metropolitan cities. At present the traffic controlling systems aresemiautomatic in nature. With the introduction of IoT in road traffic management systems, it revolutionizes the field of road traffic management system and improves the road traffic congestion problem.This paper proposes an IoT-based road traffic management system for metropolitan cities. The proposed system provides the hassle free movement of the vehicles to avoid inconvenience and reroute the higher priority vehicles. Experimental results show that the proposed system gives higher success rate for the low traffic density in the lane.
\end{abstract}

Key Words: Traffic management system, road traffic

\section{INTRODUCTION}

Road traffic is one of the major problems faced by people in metropolitan cities. As the number of vehicles is increases on the road, the major challenge is to reduce the accidents and no delay in emergency services. Manual road traffic management by traffic controller is measured as a unsafe and tedious task due to high risk of being stuck by passing vehicles and working all day even in criticalweathers conditions [1][2].

In the present scenarioincreasing road traffic is considered as aseriousproblem to be addressed. Itmay cost to life in some of the emergency situations like health issues. Therefore the situation of road traffic causesmiss happening on the roads dueto late medical help for the accidents; this will motivate us to come up with an idea to automate the road traffic management.Research into the use of automated enforcement of road traffic regulations is at an emerging stage.

One of the goals of road traffic control is reducing accident and travel time from one place to another.Reducing the time spent waiting in traffic signals.

Revised Manuscript Received on October 02, 2019.

Dr. ChandrakalaArya, School of Computing, Graphic Era Hill

University Dehradun, Uttarakhand, India

Email Id: arya.chandrakala@gmail.com

Dr. Manoj Diwakar, Department of CSE, Graphic Era (Deemed to be

University) Dehradun, Uttarakhand, India

Email Id: manoj.diwakar@gmail.com

KajalAggarwal, School of Computing, Graphic Era Hill University

Dehradun, Uttarakhand, India

Email Id: kajalaggarwal199591@ gmail.com
The Internet of Things (IoT)concept was introduced in the 1980s. IoT has become a crucial thing in the everyday lives of today's people.BroadlyIoTshave been applied in the various systems for examplewearable's, connected cars, smart cities,smart home, in agriculture field, traffic management and healthcarerespectively.Slowly and steadily IoT hasbecoming a part of almost every technology. To collect real time information IoT uses GPS and other technologies.

To make congestion free roads within metropolitan cities, it is essentialto use advanced technology for handling the problems of road traffic through gathering all the data related to the vehicles, process and evaluatethe data and find an alternative solution immediately. This paper proposes an alternative solution in real time based on the Internet of Things to solve the road traffic problems of metropolitan cities using different sensors from the junctiongenerating huge amount of data related to road traffic congestion over the given period of time and transfer to base station for further processing which helps in decision making and increasing the road capacity and efficiency.

In general, there is a need for road traffic management System for public safety and the jamming free roads which reduces accidents, and fuel consumption and improving economic profits and could provide business prospects.

With the growing population and vehicles inmetropolitan cities, the road traffic pressure on metropolitan roads is increasing. Therefore the graduallycrowded road traffic problem cannot be solved by the green transportation advancement [3]. One more problem that arises frequently is the long traffic jamming in the road reconstruction area for the expansion of current roads [4].

There are various vehicles detecting technique are present for road traffic management system such as radar, infrared, ultrasonic and microwave detectors and video traffic surveillance [5]. But these techniques are costly, inaccurate, difficult in installation and have insufficient capacitydue to these limitations are not recommended. To make the system cheaper we used the combination of sensors and IOT for automated road traffic management.

The rest of the paper is organized as follows: Section 2 presents the related work in the field of IOT based road traffic management.

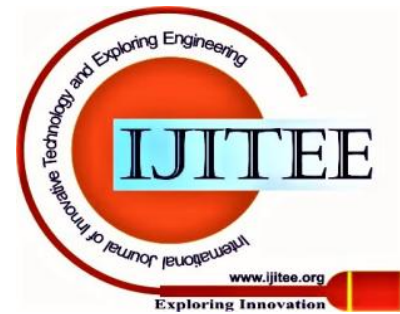




\section{IoT based Road Traffic Management System for Metropolitan Cities}

Section 3 describes our proposed architecture of the system. Result and analysis are discussed in Section 4. Finally conclusion is discussed in Section 5.

\section{RELATED WORK}

Now a day IoTis considered as the most important and challenging platform in every field. In termsof, how physical devices are interconnected in near future. The use of IOT revolutionizes the road traffic management system by redesigning the infrastructure that can solve theenlargement of the road traffic jamming problem.

In the smart cities for managing traffic IoT devices are used widely $[6,7,8,9,10]$. Rani et al. [11] proposed that IOT devices need to capture the road traffic conditions like speed, flow, and density for a particular section of road.

K Pangbourne, D Stead, M Mladenović in 2018[12] analyzing the recent concept of smart mobility referred to as Mobility as a Service (MaaS).MaaSsignifies a hybrid innovation technology which combined with a business model for conveyingcohesive access to transport services for better road traffic management.

Schimbinschi et al. [13] used the loop detectors for the analysis of traffic data using visual exploratory analysis technique. Though, with the help of loop detectors, it is not fully possible to develop moving vehicles trajectories as such sensors only detect vehicle movement but not summarize the individually recognizing vehicles capabilities.

Pinheiro [14] conducted a study on mobile phone data of metropolitan area of Rio de Janeiro, Brazil to analyze the behavior of human mobility to build human trajectories. Similar research have been conducted on the analysis of vehicle trajectory using GPS data $[15,16]$ and based on human mobility data [17].

To analyze the urban traffic,a visual analysis system known as BVisproposed by the Pei et al. in 2018. For experiment they used the comprehensivereal sparse public transportation datasets [18]. Similarly Armas et al. (2018) combined the multi-objective evolutionary algorithm with the multi-agent traffic simulator MATSim to study the level of service of urban traffic [19].For urban road networks Wang et al. (2018)proposed a prediction framework for the instant traffic flow [20].

Djahel et al. [21] proposed adaptive TMS emergency scenarios of three levels. They used a set of controllers, sensors and connected vehicle system for the changes in traffic policy system. traffic flow control usingIoT is the most prominent field for research now a days, the road traffic conditions, smart mobility service for better traffic management, loop detectors for vehicles trajectories, analysis of vehicle trajectory using GPS data, visual analysis system approaches are used to analyze the urban traffic, and adaptive TMS to analyze emergencies in road traffic etc.
On the basis of related work, the studyis summarize as

\section{PROPOSED ARCHITECTURE OF THE SYSTEM}

In our proposed system we have webcam, LED light, buzzer and RFID sensor which give the traffic jamming information. RFID sensor positioned in the four lanes near the traffic signals to collect the data from the four lanes. For the real time decision making towards road traffic management raw data comes from the sensor send to the traffic controller. Webcam, LED light, buzzer are connected to the Raspberry Pi $3 \mathrm{~B}+$ central processing unit. Sensors collected the data and then evaluated by the CPU and give the priority from the high congestion lane to the low congestion lane and take decisions. If the sensors of all the lanes give the same output status, then the same time duration will assigned bythe CPU for the corresponding lane. In our proposed algorithm we set the value of high congestion lane to 30 vehicles.

For the vehicle priority (Emergency vehicles like ambulance) XBee transmitter and receiver module operating at $2.4 \mathrm{GHz}$ frequency band are used which is connected with Raspberry Pi through Wi-Fi. XBEE will be placed on moving the higher priority vehicle and approximately $100 \mathrm{~m}$ apart from the sensor receiver antenna will be placed. When the receiving antenna receives the signals of higher priority vehicle, it will send the signals to Raspberry $\mathrm{Pi}$ and Raspberry Pi will turn LED light to red for the remaining three lanes [22]. The process of the proposed approach is shown in Figure 1.

The components of the system are described as:

\section{Raspberry Pi 3 B+}

Our proposed approach of road traffic management requires an intelligent hardware platform for better performance in the critical traffic environment. Therefore we select Raspberry Pi B+ for this purpose. It is like a credit card size motherboard of a mini computer some versions are even smaller than it. It consists of SD card instead of hard disk to install an operating system which allows convenient software updates and installation. The advantage of Raspberry $\mathrm{Pi} \mathrm{B}+$ is that after set up it can run without external peripherals. It includes GPIO pins to attach sensors, LED and other electronic hardware.

\section{Webcam}

Installed the webcam in all the traffic junctions in order to classify the higher priority vehicles and then reroute them to avoid delays.

\section{RFID Sensors}

Radio frequency identification (RFID) technology is observed as an important prerequisite of the IoT. 
RFID Sensors are arranged on each side of the road to measure the traffic flow. It refers to a technology wherereader captured the digital data via radio waves and encoded in tags which have a unique digital identity.Therefore RFID is important to connect the physical world with the digital one.

\section{Light Emitting Diode (LED) and Buzzer}

We combine buzzer with the LED for traffic light operation. Whenever a higher priority vehicle like ambulance and police vehicle stuck in the traffic jam LED turns red and buzzer sets an alarm for route diversion.

The algorithm of the proposed system is given below.

\section{Algorithm of Road Traffic Management System}

Input: RFID SENSOR (input), webcam (input)

Procedure:

\section{variables initialization}

1. Current Lane $=$ Lane I

2. Divert_Lane (I) = Lane II

3. Signal_Light $=\operatorname{Light}($ Current Lane $)$

4. Divert_Lane (II) $=$ Lane (III)

5. Divert_Lane (III) $=$ Lane (IV)

6. Divert_Lane (IV) $=$ Lane (I)

On the basis of traffic congestion selection of the traffic light status

7. Start

8. While(waiting Time $>=5 \mathrm{~min}$ )

9. if (no priority is set)

10. status of traffic light set to red

11. if(traffic congestion of Current Lane $>=30$ vehicle)

12. buzzer set an alarm

13. turn Light(CurrentLane) to green

14. Current Lane $=$ Divert_Lane (n) where $n=I$ to IV

15. If (traffic congestion of Lane $1=$ traffic congestion of Lane $2=$ traffic congestion of Lane $3=$ traffic congestion of Lane 4)

16. If (lane 1 sensor is active)

17. Wait for time $\mathrm{t}$

18. Turn light (Lane 1) to green

19. End if

20. If (lane 2 sensor is active)

21. Wait for time $\mathrm{t}$

22. Turn light (Lane 2) to green

23. End if

24. If (lane 3 sensor is active)

25. Wait for time $\mathrm{t}$

26. Turn light (Lane 3) to green

27. End if

28. If (lane 4 sensor is active)

29. Wait for time $t$
30. Turn light (Lane 4) to green

31. End if

32. End

On the basis of vehicle priority selection of the traffic light status

33. Start

34. While (waiting time $>=5 \mathrm{~min}$ )

35. If(XB sensor of current lane is high)

36. Priority of the current vehicle lane is set high

37. Turn the remaining lanes LED lights to red

38. buzzer set an alarm

39. Status of the current lane light set to green

40. Read the XB sensor of the remaining other lane until the sensor ofother lane goes high and turn red.

41. No priority set for the current lane

42. END if

43. END while

44. End

\section{RESULT AND ANALYSIS}

The implementation of IoT in road traffic management system would be helpful in smooth running of traffic. The proposed system simulated with Raspberry $\mathrm{Pi} 3 \mathrm{~B}+$ and RFID sensor. We classify vehicles as higher priority vehicles (ambulance, police vehicle, fire vehicle etc.) and no priority vehicles. In our proposed system, we used the four lanes architecture for congestion free movement of vehicles at the traffic junctions.

The overall performance of the system is defined as the hassle free journey for the people and allows priority to the emergency vehicles. The system performance is evaluated by the Repeater Operating Curve (ROC). The observational and predictive rates of success of congestion free movements of vehiclesare shown as the ascending true positive rates by the ROC curve. Table 1 show the number of vehicles passes at traffic junction without congestion at an instance of four hour period. The successful movement of vehicles from the traffic junction isconsidered as the priority and the movement times at the traffic junction. 


\section{IoT based Road Traffic Management System for Metropolitan Cities}

Table 1:Analysis of the observational and predictive probability at traffic junctions

\begin{tabular}{|l|l|l|l|l|l|l|l|}
\hline Lanes & $\begin{array}{l}\text { Vehicles at } \\
\text { the traffic } \\
\text { junction }\end{array}$ & $\begin{array}{l}\text { RFID } \\
\text { sensor }\end{array}$ & $\begin{array}{l}\text { Congestion- } \\
\text { free passes } \\
\text { in traffic } \\
\text { junction }\end{array}$ & $\begin{array}{l}\text { Observational } \\
\text { probability }\end{array}$ & $\begin{array}{l}\text { Predictive } \\
\text { probability }\end{array}$ & Success & Failure \\
\hline Lane 1 & 21 & Sensor 1 & 14 & 0.92245 & 0.93572 & 15 & 24 \\
\hline Lane 2 & 35 & Sensor 2 & 9 & 0.88656 & 0.79213 & 8 & 23 \\
\hline Lane 3 & 40 & Sensor 3 & 7 & 0.83452 & 0.86789 & 6 & 29 \\
\hline Lane 4 & 25 & Sensor 4 & 15 & 0.91743 & 0.90832 & 19 & 26 \\
\hline
\end{tabular}

From the above table 1, the frequency of the congestion free movement of vehicles at the traffic junction shows the success of the experiment.

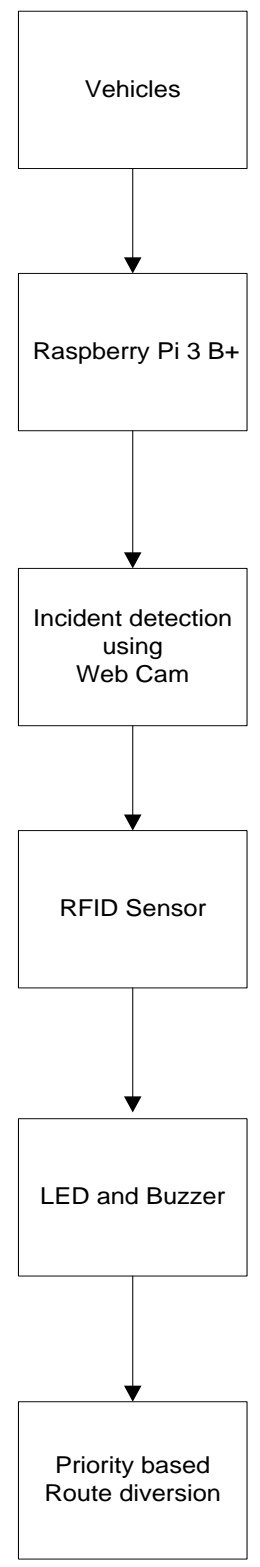

\section{CONCLUSION}

Road traffic jamming causes a significantloss in productivity and economy,as well as it highly impacted on environment. There are numerous parameters are present that mark the road traffic management and efficiency of metropolitan roads.Based on the IoT, the lane speed limit is optimized and avoids the traffic. In our proposed approach traffic is managed by collecting information from multiple RFID sensors to get the correctinformation about traffic jamming; and taking decisions accordingly, hence saving time andfuel and reducing inconvenience. It also provides an alternate route to highest priority vehicles. In future we will address the disabled and pedestrian-related challenges in road traffic congestion due to fluctuations in metropolitan traffic.

\section{REFERENCES}

1. Saravanan, S.: Implementation of efficient automatic traffic surveillance using digital image processing. In: IEEE International Conference on Computational Intelligence and Computing Research. (2014)

2. Roy, A.B., Halder, A., Sharma, R., Hegde, V.: A Novel concept of smart headphones using active noise cancellation and speech recognition. In: International Conference on Smart Technologies and Management for Computing, Communication, Controls, Energy and Materials (ICSTM), pp. 366-371. (2015)

3. F. Su, H. Dong, L. Jia, and X. Sun, “On urban road traffic state evaluation index system and method," Modern Phys. Lett. B, vol. 31, no. 01, Jan. 2017, Art. no. 1650428

4. L.-A. Gille and C. Marquis-Favre, "'Testing of urban road traffic noise annoyance modelsBased on psychoacoustic indicesUsing in situ socioacoustic survey," J. Acoust. Soc. Amer., vol. 141, no. 5, p. 3802, May 2017.

5. Stojmenovic I (2014) Fog computing: a cloud to the ground support for smart things and machine-to-machine networks. Australasian telecommunication networks and applications conference. IEEE, pp $118-122$.

6. Pan B, Zheng Y, Wilkie D, Shahabi C (2013) Crowd sensing of traffic anomalies based on human mobility and social media. In: Proceedings of the 21st ACM SIGSPATIAL international conference on advances in geographic information systems, pp 344353.https://doi.org/10.1145/2525314.2525343

7. Pinheiro CAR (2014) Human mobility behavior and predicting amount of trips based on mobile data records.

8. R. Al Mallah, A. Quintero, and B. Farooq, “Cooperative evaluation of the cause of urban traffic congestion via connected vehicles," IEEE Trans. Intell. Transp. Syst., vol. 21, no. 1, pp. 5967, Jan. 2020. 
9. Stojmenovic I (2014) Fog computing: a cloud to the ground support for smart things and machine-to-machine networks. Australasian telecommunication networks and applications conference. IEEE, pp $118-122$

10. Djahel S, Salehie Met al (2013) Adaptive traffic management for secure and efficient emergency services in smart cities. IEEE, pp 340343

11. L.P.J. Rani, M.K. Kumar, K.S. Naresh, S. Vignesh, Dynamic traffic management system usinginfrared (IR) and the internet of things (IoT), in2017 Third International Conference on ScienceTechnology Engineering \& Management (ICONSTEM), Chennai(2017), pp. 353357

12. Pangbourne, K., Stead, D., Mladenović, M., \& Milakis, D. (2018). The case of mobility as a service: A critical reflection on challenges for urban transport and mobility governance. Governance of the smart mobility transition, 33-48.

13. Schimbinschi F, Nguyen XV, Bailey J, Leckie C, Vu H, Kotagiri R (2015) Traffic forecasting in complex urban networks: Leveraging big data and machine learning. In: 2015 IEEE international conference on big data (big data), pp 1019-1024.https://doi.org/ 10.1109/BigData.2015.7363854

14. Pinheiro CAR (2014) Human mobility behavior and predicting amount of trips based on mobile data records

15. Peng C, Wong JX, Shi K-C, Lio` M (2012) Collective human mobility pattern from taxi trips in urban area. PLoS ONE 7(4):34487.https://doi.org/10.1371/journal.pone.0034487

16. Wang M, Yang S, Sun Y, Gao J (2017) Human mobility prediction from region functions with taxi trajectories. PLoS ONE 12(11):e0188735.https://doi.org/10.1371/journal.pone.0188735

17. L. Lin, C. Jiang, X.-D. Xu, Y.-L. Guo, and T. Chen, "Study on evaluation for crowd degree of trafc ow at urban tunnel access based on acceleration noise," J. Highway Transp. Res. Develop. (English Ed.), vol. 11, no. 1, pp. 7076, Mar. 2017.

18. W. Pei, Y. Wu, S. Wang, L. Xiao, H. Jiang, and A. Qayoom, "BVis: Urban traffic visual analysis based on bus sparse trajectories," J. Vis. vol. 21 , no. 5 , pp. 873883 , Oct. 2018

19. R. Armas, H. Aguirre, and K. Tanaka, "Bi-objective evolutionary optimization of level of service in urban transportation based on trafc density," Cogent Eng., vol. 5, no. 1, pp. 121, 2018.

20. W. Xiangxue, X. Lunhui, and C. Kaixun, "Data-driven short-term forecasting for urban road network trafc based on data processing and LSTMRNN," Arabian J. for Sci. Eng., vol. 44, no. 4, pp. 30433060, Apr. 2019.

21. Djahel S, Salehie Met al (2013) Adaptive traffic management for secure and efficient emergency services in smart cities. IEEE, pp 340343.

22. Joshi, A., Jain, N., \& Pandey, A. (2020). IoT-Based Traffic Management System Including Emergency Vehicle Priority. In International Conference on Intelligent Computing and Smart Communication 2019 (pp. 1501-1507). Springer, Singapore. 